\title{
Aportaciones del Modelado Paramétrico a la Toma de Decisiones: Dos Estudios de Caso desde el Proyecto de Arquitectura
}

\author{
Contributions to Decision Making from Parametric Modeling: Two Case Studies from the Architectural Project
}

\author{
Ivan Custodio dos Santos Souto \\ Noosfera Arquitetura, Brasil \\ ibanezsouto@gmail.com \\ José Nieto Martínez \\ UPCT, Spain \\ jose.nieto@upct.es
}

\author{
Matías Nieto Tolosa \\ Noosfera Arquitetura, Brasil \\ matiasnietotolosa@gmail.com \\ José Wagner García \\ Noosfera Arquitetura, Brasil \\ noosfera.projetos@terra.com.br
}

\begin{abstract}
Research progress about the contributions from parametric, generative design to the general decision-making process, specially to Design \& Decision Support Systems, into the architecture project, by 2 specific case study on professional practice in Brazil. Proposed approach deal with some contributions that contemporary media of computer graphics could add to architectural practice in a context of progressive automation, simultaneity demands and a need for higher coordination among engineering offices, taking into consideration the adaptation of handmade, industrial traditional tasks through computer-automated design, participative interfaces and digital fabrication support.
\end{abstract}

Keywords: Modelado paramétrico; Design \& Decision support systems; Ingeniería simultánea; Diseño generativo.

\section{Introducción}

El objetivo del artículo es transmitir a la comunidad académica los problemas específicos que están surgiendo en la investigación en marcha sobre Sistemas de Apoyo a la Toma de Decisión (SATD) y modelado paramétrico, como parte del trabajo dentro del estudio en torno al cual trabajan directa o indirectamente los autores, en la búsqueda de un método arquitectónico avanzado que se sirva de las posibilidades de los nuevos medios asimilando conceptos y métodos propios de esos sistemas y del pensamiento paramétrico y algorítmico, con las restricciones de dos encargos anticipados sobre necesidades reales a nivel de estudio preliminar: el primero un concurso público y el segundo un ensayo.

El grupo actual sobre SATD (Design \& Decision Support Systems, DDSS) configura una línea de investigación consolidada sobre el procedimiento y la utilidad de los sistemas de apoyo a las decisiones espaciales y formales surgidos en torno de la década de 1950 a partir de estudios teóricos sobre organizaciones en el Carnegie Institute of Technology, aplicados a la arquitectura, la planificación urbana y el ambiente construido. Está coordinado desde la Technische Universiteit Eindhoven (Holanda) como un centro internacional de investigación con participantes de Austria, Holanda, Alemania, Bélgica y Polonia, entre otros países. Partiendo de esta fundamentación teórica y de los análisis existentes sobre modelado paramétrico y SATD, se aborda el problema de cómo emplear modelos matemáticos rigurosos (una deseable inteligencia computacional) como base para tomar decisiones de proyecto que puedan mejorar el objeto arquitectónico y su comportamiento bajo condicionantes reales, así como centrar las decisiones con más énfasis en el usuario final (Timmermmans et al., 2012).

Las aportaciones de la computación evolutiva residen en su capacidad para resolver problemas exponenciales. Dentro de la progresiva automatización de tareas mecánicas y cognitivas, el aprendizaje automático (Mitchell, 1997) es un intento de automatizar algunas partes del método científico mediante métodos matemáticos sofisticados que analizan por data mining bases de información demasiado amplias como para hacerlo a mano, y en base a los cuales tomar decisiones optimizadas, predeterminadas con auxilio del cálculo. Se acepta cada vez más que el uso de modelos paramétricos probabilísticos permitirá un mejor enfoque de problemas bajo condiciones de incertidumbre y con múltiples variables en dominios estocásticos, como puede ser diseñar e instalar un hábitat en ambientes climáticos extremos. Así lo afirmaba Brunskill (2009) en su tesis de doctorado: “... the use of parametric models of the world dynamics, rewards and observations can enable efficient, provably close to optimal, decision making in large, high-dimensional uncertain environments".

\section{Metodología}

Se aborda el empleo de software de diseño paramétrico y algorítmico Grasshopper (GH) y software de modelado 3D avanzado Rhinoceros combinado con otros programas de cálculo y gestión de datos, programación basada en inteligencia artificial, interfaces digitales y software de simulación técnica (higrotérmica, 
solar, estructural e hidráulica). El modelo presentado en esta investigación incipiente da mayor relevancia al flujo de datos referentes a cada proyecto, conforme las premisas de las disciplinas complementarias (incluyendo un modelo semiótico de la información), a las posibilidades de participación del usuario y a las informaciones ambientales relacionadas con la actuación del edificio (input). La traducción y organización de los datos provenientes de las diversas fuentes en un sistema cuasiautomatizado de generación de geometrías (output) y módulos constructivos sirve como herramienta asistente en la concepción creativa del proyecto arquitectónico (Knight, 2000).

\section{Primer caso}

El primer caso de aplicación corresponde al proceso de proyecto para el concurso internacional de renovación de la Estación Antártica Comandante Ferraz promovido por el Instituto dos Arquitetos do Brasil: un proyecto de altas prestaciones constructivas, logísticas, térmicas y energéticas localizado en un ambiente extremo con aspectos simbólicos referentes a la representatividad nacional. Comienza con la definición de un lenguaje o gramática formal correspondiente a las fases del proyecto: genotipo (morfologías y reglas originales menos discutibles, ideación de un estudio preliminar), fenotipo (apariencia de las partes del modelo, correspondientes a un anteproyecto), prototipo (modelo completo casi real, simulable para el proyecto de ejecución) y estereotipo (objeto construido, con huella física). El objetivo es parametrizar el diseño para generar ' $n$ ' fenotipos discutibles y seleccionables por agentes externos, y a partir de los cuales moldear prototipos para realizar ensayos técnicos de desempeño antes de su ejecución material a escala 1:1 (Fig. 1). Las propuestas iniciales de volumetría y de componentes constructivos fueron modeladas paramétricamente en $\mathrm{GH}$. El objetivo de flexibilizar el flujo de informaciones del proyecto llevó a optar por este ambiente instrumental, donde los datos que componen la base geométrica pueden ser leídos y extraídos con facilidad, y así analizados en otro software o por otros profesionales y reincorporados al modelo, buscando una agilidad para el método proyectual y aproximándose a las ventajas de la Ingeniería Simultánea.

\section{Segundo caso}

El segundo caso corresponde a una investigación para la construcción sistemática de escuelas de bajo coste para áreas rurales y periurbanas de Brasil, afrontando el requisito de fabricar un elevado número de unidades localizadas en diferentes biomas y microclimas, abordado desde premisas de adaptabilidad, integración socio-ambiental, inteligencia morfológica y economía de medios. El diseño sistemático buscado (que podemos llamar 'semiautomático') debe vincular, mediante unas instrucciones parametrizables en un algoritmo, los datos del ambiente a una gramática constructiva basada en el ensamblaje de elementos modulares diseñados con grados de libertad controlados (variables) bajo el desafío de ser manufacturados con el auxilio de la fabricación digital de Brasil y la evolución hipotética de esta tecnología en un horizonte de 10 años, donde el usuario y el clima puedan tomar parte activa en varias etapas del proceso como agentes decisivos, sean expertos o no, con voz y voto en las decisiones de proyecto, lo que constituye de por sí una apertura metodológica cuyos resultados formales y funcionales están por explorar.

Mediante este método de proyecto se abre de forma renovada la posibilidad de integración de las informaciones recolectadas y de la participación de la comunidad relacionada en la fabricación e instalación de los componentes, pasando por la ideación de las formas del edificio y por el proceso constructivo hasta interactuar con el propio programa educativo a un nivel cognitivo y sensorial (Mitchell, 2009).

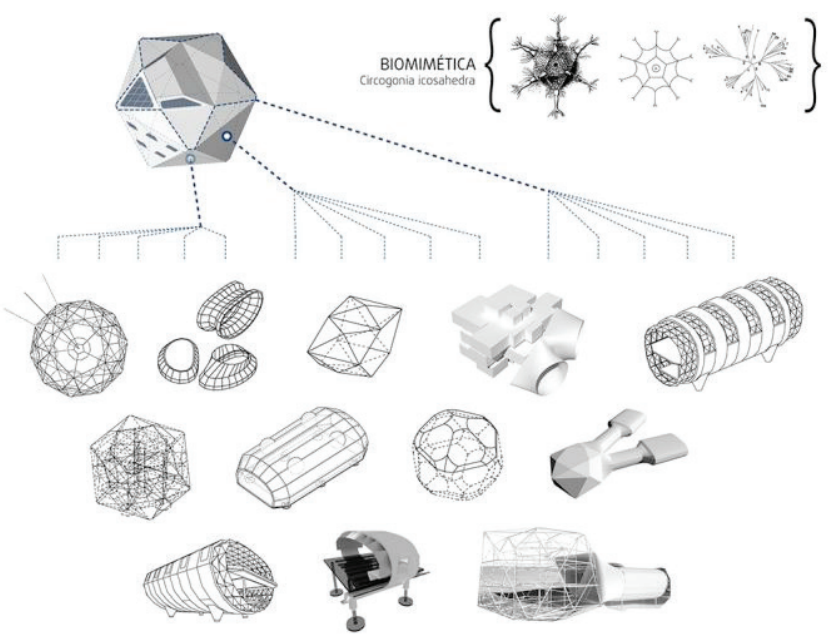

Figura 1: matriz generativa y módulos parametrizados 0 fenotipos, representando los tipos de combinaciones planteados a partir de un genotipo de proyecto (ordenación radial de flujos). En la parte superior derecha se representa el genotipo y su relación biomimética (Circogonia icosahedra, diagrama estructural y diagrama de información o árbol de decisión).

\section{Resultados}

Los resultados se centran en mostrar la operatividad de los métodos de modelado paramétrico aplicados al proceso de toma de decisiones de un proyecto arquitectónico y apuntar críticamente los aspectos problemáticos del proceso, así como algunas líneas de oportunidad abiertas.

\section{Resultados primer caso}

Se parte de una idea generadora consistente en la ordenación central de tipo radial de todos los usos y flujos energéticos, para permitir una adaptación al lugar basada en operaciones de rotación y un crecimiento modular a partir de un núcleo fijo sobre la estación existente, minimizando impactos sobre suelo antártico. Las necesidades específicas de separación de usos incompatibles y diferencias en la configuración espacial de los diversos elementos del programa llevaron a una división del proyecto en volúmenes con características específicas. Ambientes pequeños con repetición del programa y mayor compartimentación, como los camarotes y laboratorios, fueron organizados en una configuración lineal, 
permitiendo la adopción de sistemas constructivos modulares y su posible crecimiento a lo largo de los 40 años de operación previstos por la Marina de Brasil. Para la determinación y refinamiento de las soluciones posibles de proyecto referentes a la definición de los volúmenes y los componentes constructivos, algunos aspectos de desempeño fueron simulados por computador a través de $\mathrm{GH}$, ambiente en el que los modelos se desarrollan, con el software Ecotect. Creado a través del plug-in GECO, ese vínculo posibilita administrar la transferencia de la geometría modelada en Rhinoceros o en GH para análisis del desempeño ambiental y de confort en Ecotect y permite devolver los datos resultantes en tiempo real. De esta forma la simulación de absorción de la radiación solar por las superficies del cerramiento llevó a la mejora en la orientación y a la situación de los módulos en función de los valores obtenidos. También se realizó una simulación de los niveles de iluminación de los ambientes internos para diferentes formatos y tamaños de ventana, alterando el diseño de los componentes prefabricados modulares para que tuviesen el desempeño optimizado en relación a las condiciones específicas y limitadas de la luz solar en la península Antártica (menos de 4 meses al año).

El volumen propuesto para el modulo de la estación referente a las áreas de control y de uso comunitario tenía la posibilidad de albergar ambientes con disposiciones mayores y menor compartimentación $y$, por consiguiente, una organización programática más compacta, tendiendo al menor factor de forma para máxima conservación de energía y minimizando los intercambios térmicos. Esa relación fue calculada para varias configuraciones geométricas del volumen (Fig. 2).

El empleo de un modelo paramétrico fue determinante, además, en la comunicación técnica con especialistas de disciplinas complementarias (consultorías). La formación de un equipo multidisciplinar era de hecho una exigencia lógica recogida por las bases del concurso, y la dinámica de esas alteraciones fue considerada con la aplicación de un modelo paramétrico convertido de ese modo en un marco de cooperación. La adaptación dimensional de componentes estructurales en función de cambios en el proyecto se realizó mediante alteración de parámetros con el soporte de un ingeniero especialista en estructuras.

\section{Resultados segundo caso}

Este caso consistió en ensayar la parametrización de una sala de aula adaptable a partir del modelo de escuela rural tipificado según los criterios de la Fundação Nacional para o Desenvolvimento da Educação (FNDE), buscando establecer decisiones de proyecto en base a las informaciones obtenidas en tiempo real acerca de la optimización del confort ambiental para la práctica recíproca del aprendizaje y la enseñanza. A partir de una idea generadora basada en paramentos-tribuna, celosías reconfigurables y un entresuelo equipado con una biblioteca y una sala de informática elaborados a partir de una malla hexagonal irregular, se va planificando un algoritmo a partir del cual ir tomando decisiones de proyecto para aproximarse a una panoplia de soluciones que cumplan los objetivos predeterminados sin perder el carácter adaptativo deseable.

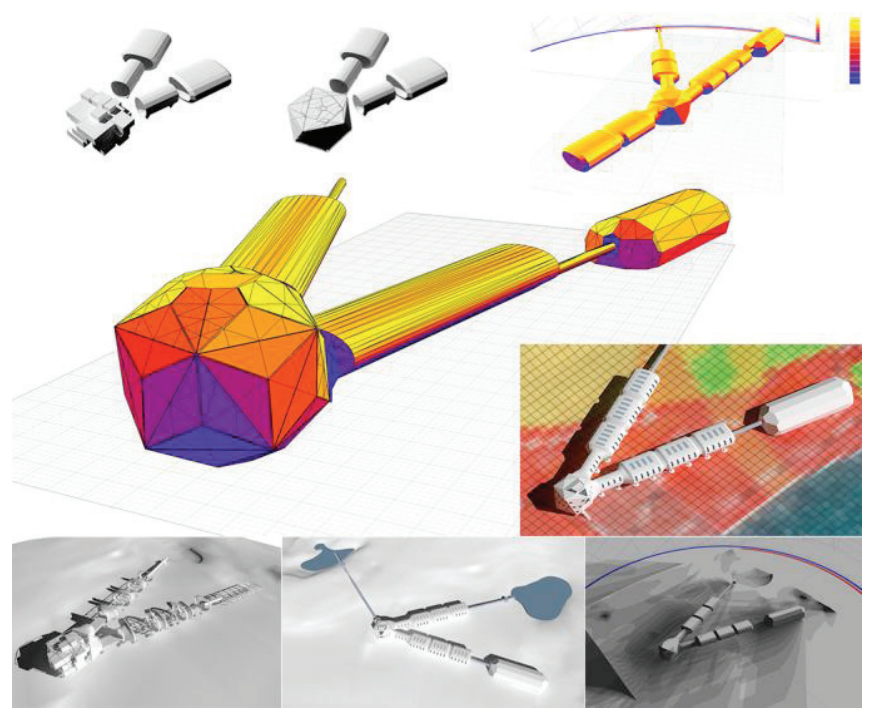

Figura 2: simulaciones y modelos paramétricos de la estación analizados en tiempo real bajo diversos criterios de desempeño.

Haciendo un inciso desde una perspectiva transdisciplinar, Medeiros \& Garcia (2011), a propósito del impacto de una concepción semiótica del pensamiento y el lenguaje en la producción de proyectos arquitectónicos mediados o compartidos en programas digitales, declaraban: "en el campo del mundo digital, la arquitectura ya está dando un paso evolutivo más en su matriz conceptual, con la aparición de programas de ideación o prospección de soluciones arquitectónicas en colaboración continua con recursos de la Inteligencia Artificial, en programas o sistemas computacionales especialistas. Avanza más allá de un proceso de visualización científica - que en rigor crea posibilidades más precisas de percepción de formas y cualidades tridimensionales- con la utilización de recursos gráficos por computador que operan e interfieren en el núcleo del proceso de creación arquitectónica. La máquina computacional lado a lado con el arquitecto".

El proceso computacional realizado fue un test en $\mathrm{C}++$, redactado en plataforma Linux, de todas las combinaciones posibles de los grados de libertad disponibilizados para el proyecto, anticipando algunos aspectos deseables para el comportamiento posterior del modelo. En cada combinación, fueron evaluados los siguientes Indicadores de Desempeño (ID): la viabilidad geométrica, el volumen máximo, el confort acústico, la pendiente de la cubierta y la resistencia estructural. Las combinaciones (especies) que no representaban valores adecuados en todos los ID fueron descartadas, en una especie de selección biológica. Se computaron 11.793.360 testes, de los cuales apenas 9.061 presentaron valores satisfactorios en todos los ID (Fig. 3). Esas soluciones fueron posteriormente introducidas en $\mathrm{GH}$ para elegir las alternativas a utilizar en la siguiente fase de diseño. 


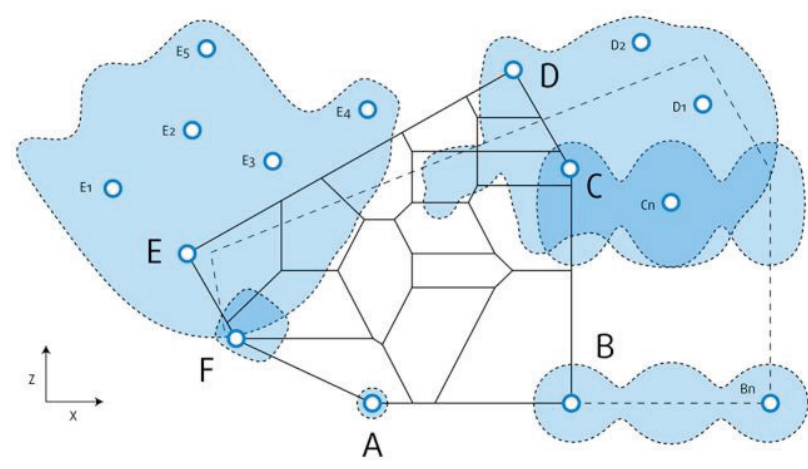

Figura 3: se representa sombreado el campo o espacio de soluciones de las combinaciones válidas computadas tal que el tiempo de reverberación fuese de 0.4 a 0.6 segundos, el volumen de la sala fuese menor de $350 \mathrm{~m}^{3}$, la inclinación de la cubierta fuese mayor o igual al $2 \%$ y la viga de cubierta tuviese una luz menor o igual a $4 \mathrm{~m}$. La sección ABCDEF representada es sólo una de las posibles.

En su tesis de doctorado, Davis (2013) analiza las 5 críticas al 'parametrismo' ${ }^{1}$, afirmando que (1) el modelado paramétrico requiere un cierto grado de front-loading, de planificación con una lógica parecida a la de la programación de software, (2) anticipar la flexibilidad de un modelo puede resultar de una dificultad inesperada, (3) grandes cambios rompen incluso un modelo paramétrico de gran flexibilidad, (4) los cambios pueden resultar visualmente poco reconocibles y (5) reutilizar y compartir modelos puede ser problemático. A partir de aquí, Davis apunta algunos retos actuales del modelado paramétrico, tema ausente en la mayoría de discursos arquitectónicos contemporáneos, como dar mucha más relevancia a la planificación buscando anticipar fases del diseño, anticipar la propia flexibilidad del modelo pensando en su uso compartido por otros agentes y la posibilidad de cambios o asumir el papel limitado de un modelo frente procesos que implican cambios críticos, estructurales o de ideación (del genoma) parametrizando incluso los conceptos generadores. Resumiendo, aconseja dedicar más esfuerzos a planificar la propia programación.

\section{Reflexiones}

Este artículo se completa en la medida que estimula o abre un análisis de la necesaria adaptación del papel del arquitecto contemporáneo como director de una orquesta constructiva que incorpora de forma exponencial procesos e instrumentos automatizados así como un lenguaje propio de las ciencias de la complejidad (con una terminología biológica cada vez más común en la teoría de la arquitectura y el urbanismo desde las aportaciones de Luhmann o von Bertlanffy) con una creciente exigencia de transparencia en los procesos productivos. Además, cabe resaltar las diferencias conceptuales e instrumentales entre proyectar y programar que a menudo se confunden, la importancia que toma la emergente arquitectura de la información y la

1 Tomado de Smith, Rick (2007). "Technical Notes From Experiences and Studies in Using Parametric and BIM Architectural Software." Published 4 March. http://www.vbtllc.com/images/VBTTechnicalNotes.pdf apertura selectiva de la toma de decisiones a agentes no expertos. Este último tema conlleva la importancia de poder dar voz a agentes de decisión potencial generalmente silenciosos (o silenciados) pero que son afectados en gran medida por el estereotipo: clima, biocenosis y especialmente humanos no expertos, utilizando automatismos como intérpretes de esas realidades y sus intereses, para después convenir una capacidad ponderada de voto. Por último, resaltar que los datos no tienen por qué venir únicamente ya dados para elaborar un proyecto, sino que los datos mismos pueden ser parte y objeto del propio diseño (tenemos el ejemplo del proyecto Authored Sensing sobre el concepto 'designed datastreams', expuesto en SmartGeometry 2011 por Steinfeld, Park \& Novelli), criticando el carácter determinista existente en la predeterminación de comportamientos matemáticos y materiales mediante protocolos y algoritmos de programación y su pretendida objetividad absoluta: también interesa la conciencia que subyace a una automatización parcial de los procesos creativos.

\section{Agradecimientos}

Este trabajo ha sido posible gracias al formidable trabajo del Colectivo PKMN Architectures (www.pkmn.es) como co-autores en el desarrollo y definición del proyecto para el concurso Estación Antártica Comandante Ferraz; así como ha sido mucho más asequible gracias a las aportaciones del ingeniero Yopanan C.P. Rebello y Thermoplan Ltda. (asesorías estructural y térmica, respectivamente, para el primer caso), el constructor Helio Olga Jr. (asesoría en cálculo y construcción en madera para el segundo caso) y a Ricardo Maciel Gazoni (www.semiotic.com.br), mestrando em Tecnologias da Inteligência e Design Digital - TIDD / PUC-SP y socio fundador de Semiotic Systems Ltda. (asesoría en la programación en $\mathrm{C}++$ para el segundo caso).

\section{Referencias}

Brunskill, E.P. (2009). Compact parametric models for efficient sequential decision making in high-dimensional, uncertain domains. Ph.D. Thesis, Dep. Of Electrical Engineering and Computer Science, MIT.

Davis, D. (2013). Modelled on Software Egineering: Flexible Parametric Models in the Practice of Architecture. Ph.D. Thesis, School of Architecture and Design, RMIT University.

Knight, T. W. (2000). Shape grammars in education and practice: history and prospects. International Journal of Design Computing. Sydney: 1999-2000.

Medeiros, R., Garcia, J. (2011). Arquitetura Semiótica - Objeto imediato, objeto dinâmico e a matriz da linguagem. 14a Jornada Peirceana, 8191.

Mitchell, T. (1997). Machine Learning. McGraw Hill.

Mitchell, W.J. (2009). A lógica da arquitetura. Campinas: Editora da UNICAMP.

Timmermmans, H.J.P. et al. (2012). Prototype evaluation of a user centered design system for smart homes. In: Design and Decision Support Systems in Architecture and Urban Planning Conference (DDSS2012), 27-29, August 2012, Eindhoven, the Netherlands, 2012. 